\title{
A Novel Downward-Looking Linear Array SAR Imaging Method Based on Multiple Measurement Vector Model with $L_{2,1}$-Norm
}

\author{
Le Kang $\mathbb{D}^{1,2}$ Tian-chi Sun $\mathbb{D},,^{1,2}$ Jia-cheng Ni $\mathbb{D}^{1,2}$ Qun Zhang $\mathbb{D},{ }^{1,2,3}$ and Ying Luo $\mathbb{D}^{1,2}$ \\ ${ }^{1}$ Information and Navigation College, Air Force Engineering University, 710077, China \\ ${ }^{2}$ Collaborative Innovation Center of Information Sensing and Understanding, 710077, China \\ ${ }^{3}$ Key Laboratory for Information Science of Electromagnetic Waves (Ministry of Education), Fudan University, 200433, China
}

Correspondence should be addressed to Jia-cheng Ni; littlenjc@sina.com

Received 28 April 2021; Accepted 17 November 2021; Published 14 December 2021

Academic Editor: Biswajeet Pradhan

Copyright (C) 2021 Le Kang et al. This is an open access article distributed under the Creative Commons Attribution License, which permits unrestricted use, distribution, and reproduction in any medium, provided the original work is properly cited.

Downward-looking linear array synthetic aperture radar (DLLA SAR) is a kind of three-dimensional (3-D) radar imaging system. To obtain the superresolution along the crosstrack direction of DLLA SAR, the sparse regularization models with single measurement vector (SMV) have been widely applied. However, the robustness of the sparse regularization models with SMV is unsatisfactory, especially in the low signal-to-noise rate (SNR) environment. To solve this problem, we proposed a novel imaging method for DLLA SAR based on the multiple measurement vector (MMV) model with $L_{2,1}$-norm. At first, we exchange the processing order between the along-track (AT) domain and the crosstrack (CT) domain to keep the same sparse structure of the signal in the crosstrack domain so that we can establish the imaging problem as a sparse regularization model based on the MMV model. Moreover, the mixed $L_{2,1}$-norm is introduced into the regularization term of the MMV model. Finally, the modified orthogonal matching pursuit (OMP) algorithm is designed for the MMV model with the $L_{2,1}$-norm. The simulations verify that the proposed method has better performance in the lower SNR environment and requires lower computation compared with the conventional methods.

\section{Introduction}

Synthetic aperture radar (SAR) has been widely applied in the field of remote sensing to obtain radar images without being affected by weather, daylight, or cloud coverage $[1,2]$. Over the past decades, three-dimensional (3-D) SAR has been a development trend of SAR since it can generate 3-D radar images, which can provide more information for remote sensing. The typical 3-D SAR systems include circular SAR (CSAR) [3], tomographic SAR (TomoSAR) [4], and array SAR [5]. Downward-looking linear array (DLLA) SAR generates 3-D radar images by using a linear array along the wings to illuminate the overflown scene $[6,7]$. By modifying the traditional SAR imaging algorithms, 3-D imaging algorithms for DLLA SAR based on range-Doppler (RD) [8], chirp scaling [9], and polar formatting [10] are proposed. For these imaging algorithms, the resolutions in range, along-track (AT), and crosstrack (CT) directions are obtained by pulse compression, synthetic aperture, and beam-forming, respectively. As a result, the CT resolution is mainly determined by the length of the linear array along the CT direction, which leads to poor resolution in the CT direction since the length of the linear array is limited by the platform.

In the compressed sensing (CS) theory, the sparse signals can be exactly recovered with a high probability from the low-dimensional measurement signal $[11,12]$. Since the scatters in the imaging scene are generally sparse in the 3-D space, the sparsity of DLLA SAR echo holds in the spatial domain. As a result, CS are introduced to CT compression of DLLA SAR in recent years [13-21]. In [13], the signal processing in the CT domain is transformed into the sparse reconstruction with $L_{0}$ regularization from noisy measurements. Considering the nonuniform linear array, the CS method based on truncated singular value decomposition (SVD) is introduced to the processing of the CT domain to achieve superresolution [14]. For the airborne DLLA SAR with a sparse linear array, an $L_{1}$-norm-based 


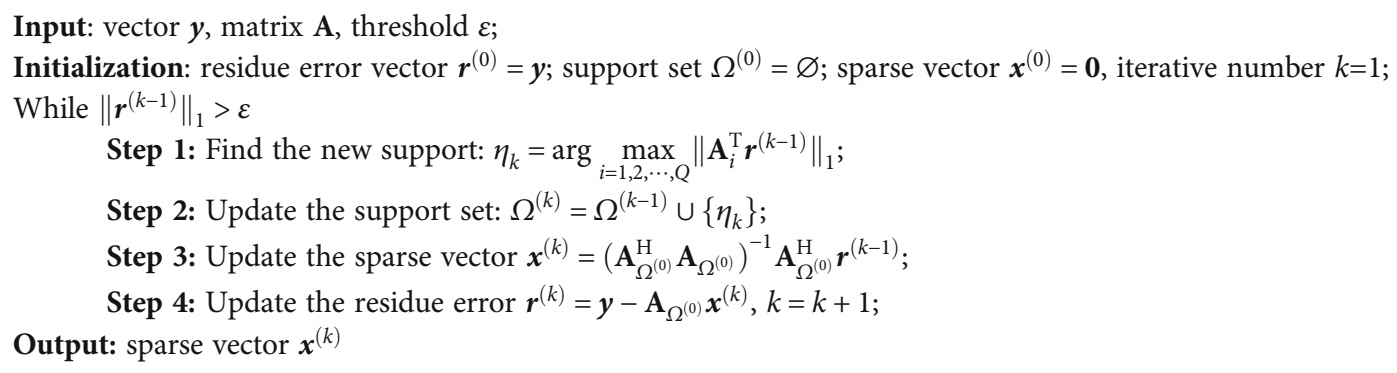

Algorithm 1: SMV-OMP algorithm.

DLLA SAR imaging method is proposed by combining the polar formatting. To solve the matrix mismatch problem, the processing in the CT domain is transformed into the atomic-norm-based CS reconstruction [16-19]. However, the CT compression of the above methods is realized by the single measurement vector (SMV) model. Although these SMV-based methods can achieve better resolution and lower sidelobes in the CT domain, the robustness of the sparse regularization models with the SMV model is unsatisfactory, especially in the low signal-to-noise rate (SNR) environment [12]. Compared with the classical single measurement vector (SMV) model, the multiple measurement vector (MMV) model in CS theory has stronger antinoise performance and lower computation complexity by utilizing the same sparse structures of multiple measurement signals [20]. The MMV model has been successfully introduced into 2-D SAR imaging since the sparse structures of different pulses are the same in SAR measurements $[21,22]$. The different pulses in SAR are equal to the different AT sampling points in DLLA SAR, so the MMV model has been directly utilized in the AT domain [23]. However, the CT sparse structures of different pulses are different so that the MMV model cannot be directly utilized in the CT domain.

In this paper, a novel imaging method for DLLA 3D SAR based on the MMV model and a mixed matrix norm is proposed. At first, we construct the same CT sparse structure of different pulses by exchanging the processing sequence of $\mathrm{CT}$ and AT domain so that the imaging problem can be regarded as a sparse regularization model based on the MMV model. Moreover, the mixed $L_{2,1}$-norm is introduced into the regularization term of the MMV model. Finally, the proposed imaging model is solved by the modified MMV orthogonal matching pursuit (MMV-OMP) algorithm. The proposed method can obtain the CT superresolution in the lower SNR environment and require lower computation compared with the conventional methods, which is verified by simulation experiments.

\section{Reviews of the SMV Model and MMV Model}

In CS theory, the signal $s \in \mathbb{C}^{Q}$ is sparse if the sparse level $K$, the number of the nonzero values in $\mathbf{s}$, is much less than $Q$. For a downsampling matrix $\Phi \in \mathbb{C}^{N \times Q}, N<Q$, the lowdimensional measurement vector of $\mathbf{s}$ can be written as $\mathbf{y}=\boldsymbol{\Phi} \mathbf{s}, \mathbf{y} \in \mathbb{C}^{N \times 1}$. The sparse representation of $\mathbf{s}$ can be written as $\mathbf{s}=\Psi \mathbf{x}$, where $\mathbf{x}$ is sparse and $\boldsymbol{\Psi}$ is the sparse basis matrix. (When $\mathbf{s}$ is sparse, the sparse basis matrix $\Psi$ is the unit matrix.) If the measurement matrix $\mathrm{A}=\boldsymbol{\Phi} \Psi$ satisfies the Restricted Incoherence Property (RIP), the problem, which is to solve the sparse signal $\mathbf{x}$ on the condition that the $\mathbf{y}$ and $\mathbf{A}$ are known, can be transformed as the 1-norm minimization [11]:

$$
\begin{aligned}
& \min \|\mathbf{x}\|_{1} \\
& \text { s.t. } \quad \mathbf{y}-\mathbf{A x}<\boldsymbol{\varepsilon},
\end{aligned}
$$

where $\|\cdot\|_{1}$ is the 1 -norm operator and $\varepsilon$ is a value related to the noise.

When the signal $\mathbf{y}$ is a vector, $\mathbf{y}$ is called the single measurement signal, and the model in Equation (1) is called the SMV model. The SMV model can be solved by many CS reconstruction algorithms. Here, we briefly introduce the OMP algorithm [24] in Algorithm 1, where ${ }^{\mathrm{T}}$ is the matrix transposition, ${ }^{(k)}$ is the $k$ th iteration, $\|\cdot\|_{1}$ is the $l_{1}$-norm, the support set $\Omega$ is the index set of nonzero values, and $\mathbf{A}_{\Omega}$ is formed by the columns of $\mathbf{A}$ with the index in $\Omega$.

For $L$ single measurement signals $\mathbf{y}_{1}, \mathbf{y}_{2}, \cdots, \mathbf{y}_{L}$ with the same measurement matrix $\mathbf{A}$, the SMV model in Equation (1) has to be solved $L$ times to obtain the corresponding sparse signals $\mathbf{x}_{1}, \mathbf{x}_{2}, \cdots, \mathbf{x}_{L}$. However, if the sparse structures of $\mathbf{x}_{1}, \mathbf{x}_{2}, \cdots, \mathbf{x}_{L}$ are the same, which means that the positions of the nonzero values in $\mathbf{x}_{1}, \mathbf{x}_{2}, \cdots, \mathbf{x}_{L}$ are the same, the MMV model can be expressed as [20]

$$
\begin{aligned}
& \min \|\mathbf{X}\|_{1} \\
& \text { s.t. } \quad \mathbf{Y}-\mathbf{A X}<\boldsymbol{\varepsilon},
\end{aligned}
$$

where $\mathbf{Y}=\left[\mathbf{y}_{1}, \mathbf{y}_{2}, \cdots, \mathbf{y}_{L}\right]$ and $\mathbf{X}=\left[\mathbf{x}_{1}, \mathbf{x}_{2}, \cdots, \mathbf{x}_{L}\right]$. In the MMV model, $\mathbf{X}$ can be obtained by solving Equation (2) only once, so that the MMV model has lower complexity and better antinoise performance compared with the SMV model.

\section{SMV-Based DLLA SAR Imaging Procedure}

The geometry of DLLA SAR is shown in Figure 1. The radar platform flies along the $X$-axis. The velocity is $v$, and the height is $H . N$ antenna elements are distributed under the wings, and the interval space between every two elements is $d$. The length of the linear array is $L_{y}=(N-1) d$. $O$ is 


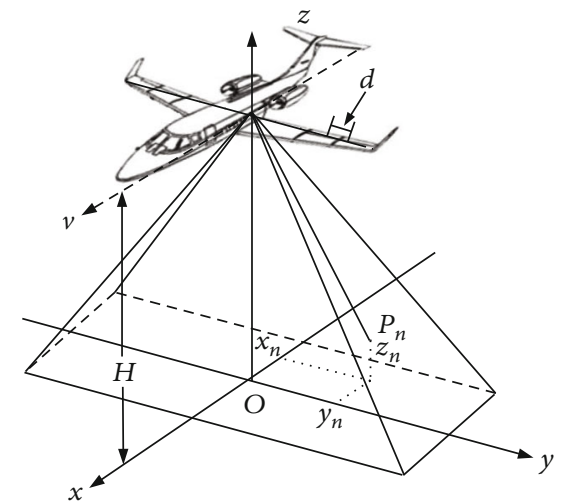

Figure 1: Geometry of DLLA 3-D SAR. The radar platform flies along the $X$-axis with velocity $v$ at height $H$. The linear array contains $N$ antenna elements equally spaced with the distance $d$, which is distributed along the crosstrack direction.

the imaging center. The position of the $n$th antenna element of the linear array is $\left(x_{m}, y_{n}, H\right)$ in the coordinate system.

Suppose that the antenna elements transmit the linear frequency modulation (LFM) signal, the echo signal of the $n$th antenna element can be represented as

$$
\begin{aligned}
S\left(t, t_{m}, y_{n}\right)= & \sum_{K} \sigma_{k} \operatorname{rect}\left(\frac{t-2 R\left(t_{m}, y_{n}\right) / c}{T_{r}}\right) \\
& \cdot \exp \left(j \pi K_{r}\left(t-\frac{2 R\left(t_{m}, y_{n}\right)}{c}\right)^{2}-j \frac{4 \pi}{\lambda} R\left(t_{m}, y_{n}\right)\right),
\end{aligned}
$$

where $t$ denotes the fast time, $t_{m}$ denotes the slow-time, $\operatorname{rect}(\cdot)$ is the unit rectangular function, $\sigma_{k}$ is the scattering coefficient of the $k$ th scattering point, $c$ is the light speed, $K_{r}$ is the chirp rate, $\lambda$ is the wavelength, $T_{r}$ is the pulse width, $e$ is the noise, and $R\left(t_{m}, y_{n}\right)$ is the instantaneous distance between the $n$th antenna element and the point scatterer $P_{k}\left(x_{k}, y_{k}, z_{k}\right)$ at the slow time $t_{m}$, which can be written as

$$
\begin{aligned}
R\left(t_{m}, y_{n}\right) & =\sqrt{\left(v t_{m}-x_{k}\right)^{2}+\left(y_{n}-y_{k}\right)^{2}+\left(H-z_{k}\right)^{2}} \\
& \approx R_{0}+\frac{\left(v t_{m}-x_{k}\right)^{2}}{2 R_{0}}+\frac{y_{n}^{2}-2 y_{n} y_{k}}{2 R_{0}}
\end{aligned}
$$

where $R_{0}=\sqrt{x_{k}^{2}+y_{k}^{2}+\left(H-z_{k}\right)^{2}}$.

The imaging procedure of SMV-based DLLA SAR is shown in Figure 2, which contains three steps: range compression, AT compression, and CT compression. At first, the echo signal of Equation (3) is discretized into a 3-D matrix, where $N_{\mathrm{r}}, M$, and $N$ are the sampling numbers in the range (fast time) domain, AT (slow time) domain, and CT domain, respectively.
The range compression is realized by range-matched filtering and range migration correction. After the range compression, the 3-D signal has been focused in the range domain and can be regarded as $N_{\mathrm{r}}$ range slices. Then, the AT compression is realized by the AT matched filtering for each range slice of the 3-D signal. After the AT compression, the signal of each range slice has been focused on the AT domain. In the cross-along-track plane, each line represents the signal vector with the same positions in the range and AT directions. The black lines of the cross-along-track plane mean that the scattering points exist in the corresponding positions in the range and AT directions.

For each line in the cross-along-track plane, the length of the original vector is the CT sampling number $N$. To obtain the CT superresolution, the imaging scene is discretized by $Q(Q>N)$ grids in the CT directions. The signal processing of each line in the cross-along-track plane is transformed into an SMV model, in which the original signal of each line is regarded as the measurement signal $\mathbf{y}$ and each line after the CT compression is regarded as the sparse signal $\mathbf{x}$. After the CT compression, the sparse structures of the lines in the cross-along-track plane are different because they are determined by the positions of the scattering points. As a result, the condition of the MMV model is not satisfied so that the $M$ lines in each range slice have to be solved by the SMV model independently.

3.1. Proposed DLLA SAR Imaging Method. Since only a few scatterers are presented in the same range cell, the sparsity of the echo signal is satisfied for DLLA SAR imaging. To introduce the MMV model into the CT domain of DLLA SAR, we exchange the processing sequence of the CT compression and the AT compression to construct the same sparse structure after the CT compression. The proposed imaging procedure of MMV-based DLLA SAR is shown in Figure 3, which contains range compression, CT compression, and AT compression.

3.2. Range Compression. The range compression of the proposed method is the same as that of the traditional methods. The details of the range compression in DLLA SAR can be found in [25]. Firstly, the echo signal should multiply by the range-matched filter function and the range migration correction functions in the frequency domain. Then, the signal in the frequency domain should be transformed back into the time domain and it can be written as

$$
\begin{aligned}
S_{r}\left(t, t_{m}, y_{n}\right)= & \sum_{K} \sigma_{k} \operatorname{sinc}\left(B_{r}\left(t-\frac{2 R_{0}}{c}\right)\right) \\
& \cdot \exp \left(-j \frac{4 \pi}{\lambda}\left(R_{0}+\frac{\left(v t_{m}-x_{k}\right)^{2}}{2 R_{0}}+\frac{y_{n}^{2}-2 y_{n} y_{k}}{2 R_{0}}\right)\right),
\end{aligned}
$$

where $B_{r}=T_{r} K_{r}$ is the range bandwidth and $R_{0}$ and $y_{n}$ can be obtained before imaging because the imaging geometry 


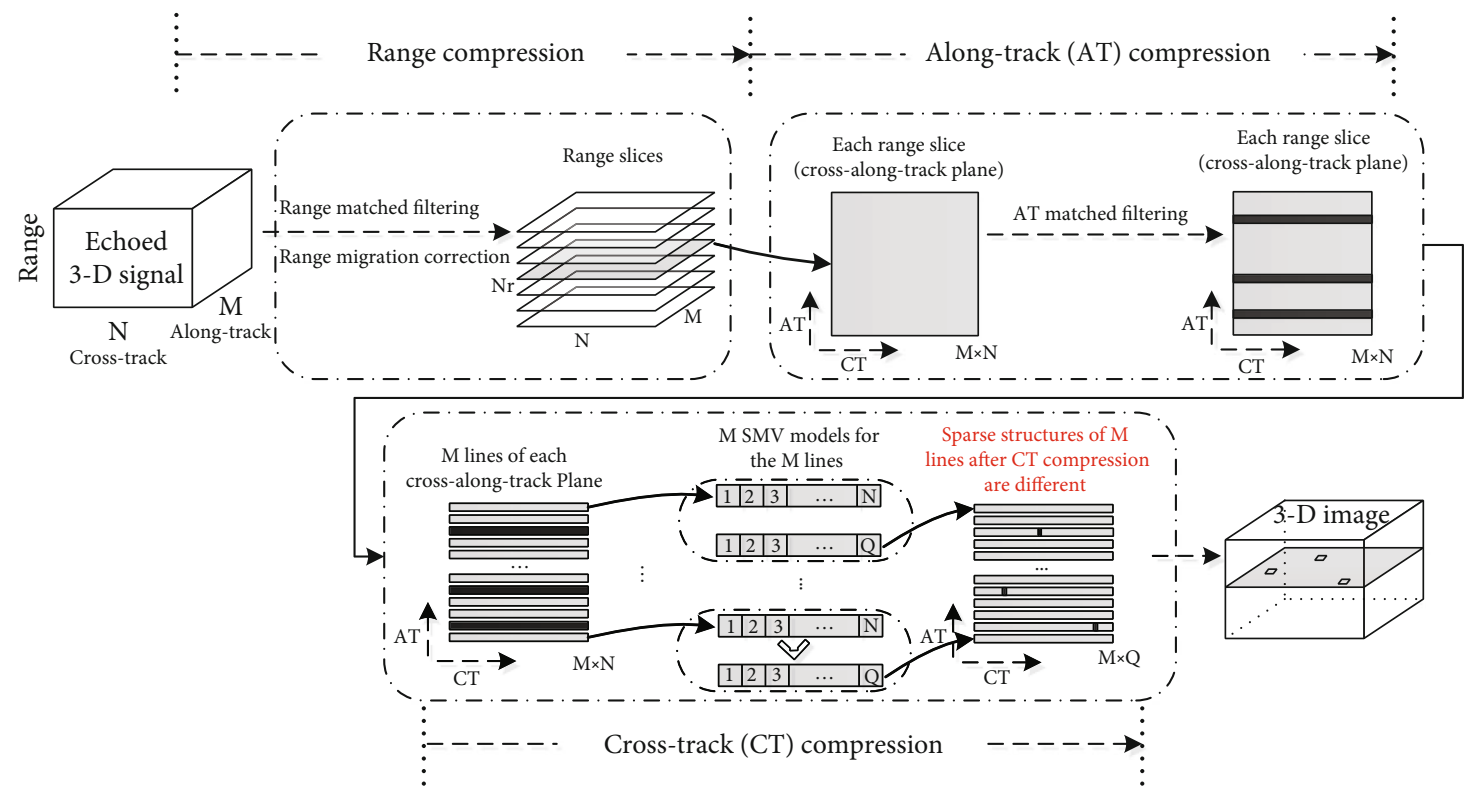

FIGURE 2: Imaging procedure of SMV-based DLLA 3-D SAR.

is known. As a result, the signal after compensating the phase term only related with $R_{0}$ and $y_{n}$ is [25]

$$
\begin{aligned}
S_{r}\left(t, t_{m}, y_{n}\right)= & \sum_{K} \sigma_{k} \operatorname{sinc}\left(B_{r}\left(t-\frac{2 R_{0}}{c}\right)\right) \\
& \cdot \exp \left(-j \frac{2 \pi\left(v t_{m}-x_{k}\right)^{2}}{\lambda R_{0}}\right) \exp \left(-j \frac{4 \pi y_{n} y_{k}}{\lambda R_{0}}\right) .
\end{aligned}
$$

3.3. Crosstrack Compression by the $M M V$ Model with $L_{2,1^{-}}$ Norm. After range compression, each measurement vector is a line along the CT direction so that each range slice contains $M$ measurement vectors. Since the CT compression is before the AT compression, the signal of a range slice focuses in several lines along the AT direction, which means that the $M$ measurement vectors after CT compression have the same sparse structure. As a result, the MMV model can be introduced into the CT compression.

The imaging scene is discretized as equal grids $\widehat{\mathbf{y}}=$ $\left[y \wedge_{1} ; \cdots ; y \wedge_{N}\right]^{T} \in \mathbb{C}^{N \times 1}$ along the CT direction. The measurement signal of the $m$ th pulse can be expressed as $\mathbf{s}_{m}=$ $\left[s\left(t_{m}, y_{1}\right) ; \cdots ; s\left(t_{m}, y_{N}\right)\right]^{T} \in \mathbb{C}^{N \times 1}$. Suppose the corresponding focused signal after crosstrack compression is $\mathbf{g}_{m}=$ $\left[g_{1}\left(t_{m}\right) ; \cdots ; g_{N}\left(t_{m}\right)\right]^{T} \in \mathbb{C}^{N \times 1}$. Therefore, the echo signal of all CT sampling points at slow-time $t_{m}$ can be written as

$$
\begin{aligned}
\mathbf{s}_{m} & =\left[\varphi_{1}, \varphi_{2}, \cdots, \varphi_{N}\right]\left[g_{1}\left(t_{m}\right), g_{2}\left(t_{m}\right), \cdots, g_{N}\left(t_{m}\right)\right]^{T} \\
& =\Psi \mathbf{g}_{m}, \quad m=1,2, \cdots M,
\end{aligned}
$$

where $\Psi=\left[\varphi_{1}, \varphi_{2}, \cdots, \varphi_{N}\right]$ is the sparse basis matrix. The $n$th column of the sparse basis matrix $\Psi$ can be expressed as

$$
\varphi_{n}=\left[\exp \left(-j \frac{4 \pi}{\lambda R_{0}} y_{1} y \wedge_{n}\right), \exp \left(-j \frac{4 \pi}{\lambda R_{0}} y_{2} y \wedge_{n}\right), \cdots, \exp \left(-j \frac{4 \pi}{\lambda R_{0}} y_{N} y \wedge_{n}\right)\right]^{T}
$$

Suppose that the downsampling matrix is $\Phi_{m} \in \mathbb{R}^{N^{\prime} \times N}$, which is formed by the random $N^{\prime}$ columns of an $N$-by- $N$ identity matrix. Thus, the matrix form of the downsampled signal is

$$
\mathbf{s}_{m}^{\prime}=\mathbf{A g}_{m}
$$

where $m=1,2, \cdots M, \mathbf{A}=\boldsymbol{\Phi}_{m} \boldsymbol{\Psi}$. The sample rate (SR) is defined as $N^{\prime} / N$.

The model in Equation (9) is an SMV model, in which the sparse vector $\mathbf{g}_{m}$ can be solved by the OMP algorithm. For the linear array, the CT compression of each signal of any antenna element needs to solve an SMV model. As a result, we should solve $M$ SMV models to get the crosstrack 


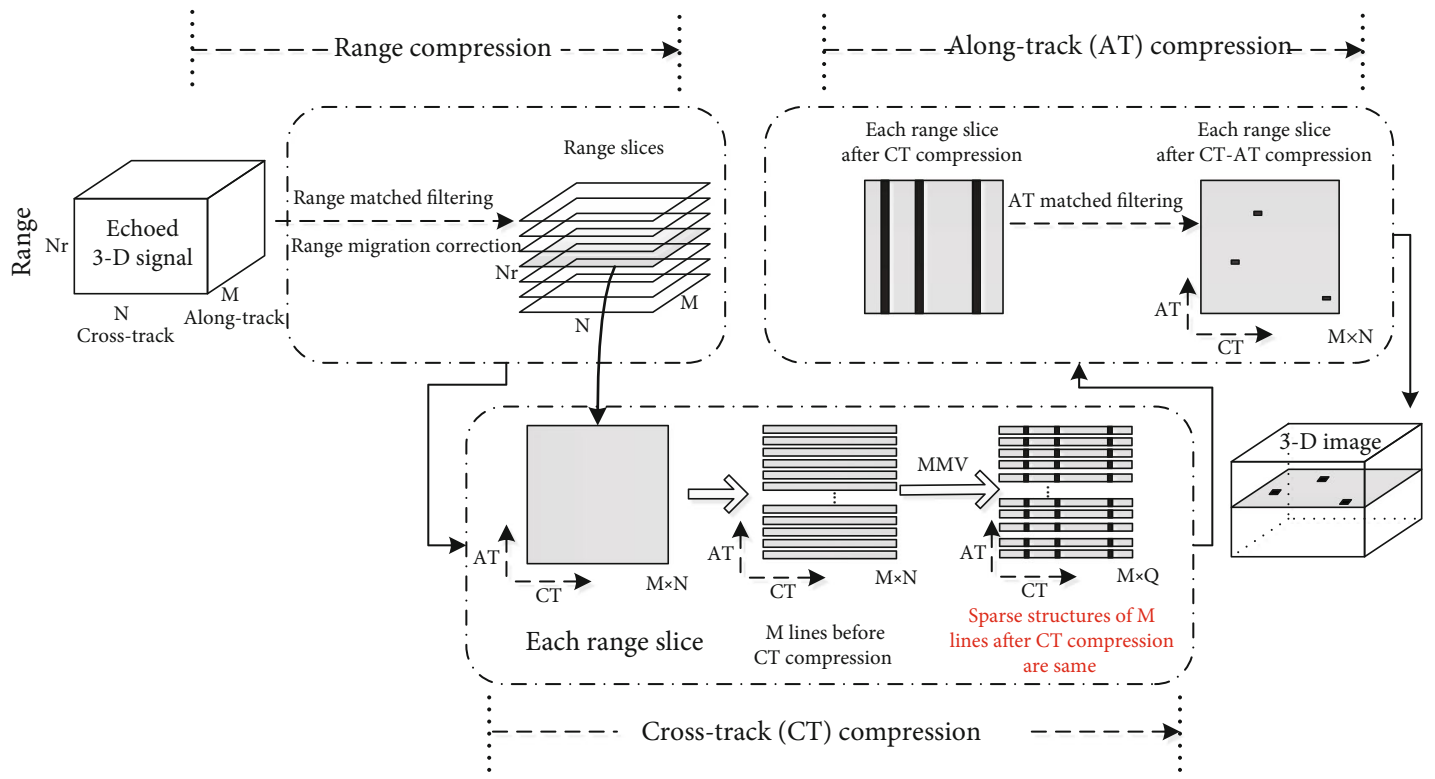

FIGURE 3: Imaging procedure of MMV-based DLLA 3-D SAR.

Input: matrix $\boldsymbol{\Xi}$, matrix $\mathbf{A}$, threshold $\varepsilon$;

Initialization: residue error matrix $\mathbf{R}^{(0)}=\boldsymbol{\Xi}$, support set $\Omega^{(0)}=\varnothing$, sparse matrix $\mathbf{G}^{(0)}=\mathbf{0}$, iterative number $k=1$; While $\left\|\mathbf{R}^{(k-1)}\right\|_{2,1}>\varepsilon$

Step 1: Find the new support: $\eta_{k}=\arg \max _{i=1,2, \cdots, Q}\left\|\mathbf{A}_{i}^{\mathrm{T}} \mathbf{R}^{(k-1)}\right\|_{2,1}$;

Step 2: Update the support set: $\Omega^{(k)}=\Omega^{(k-1)} \cup\left\{\eta_{k}\right\}$;

Step 3: Update the sparse matrix $\mathbf{G}^{(k)}=\left(\mathbf{A}_{\Omega^{(k)}}^{\mathrm{H}} \mathbf{A}_{\Omega^{(k)}}+(\lambda / 2) \boldsymbol{\Lambda}\left(\mathbf{G}^{(k-1)}\right)\right)^{-1} \mathbf{A}_{\Omega^{(k)}}^{\mathrm{H}} \mathbf{R}^{(k-1)}$;

Step 4: Update the residue error $\mathbf{R}^{(k)}=\boldsymbol{\Xi}-\mathbf{A}_{\Omega^{(k)}} \mathbf{R}^{(k-1)}, k=k+1$;

Output: sparse vector $\mathbf{G}^{(k)}$

Algorithm 2: MMV-OMP algorithm based on the $L_{2,1}$-norm.

recovery signal of one range slice. Assuming the range sampling number is $N_{r}$, the number of SMV recovery times is $M \cdot N_{\mathrm{r}}$ to obtain the $3-\mathrm{D}$ imaging result. Since the CS reconstruction algorithm is much more complex than the matched filtering, the SMV-based DLLA SAR imaging methods generally have high computational complexity.

For the MMV model, each measurement vector should have the same sparse structure. As shown in Figure 2, this condition cannot be satisfied if the echo signal is handled in the AT domain before the CT domain, because the CTfocused results are points in each range slice, which means the sparse structures are different in different AT measurement vectors. However, as shown in Figure 3, the CT compression is before the AT compression in the proposed imaging flow process. Meanwhile, after range compression and CT compression, the focused results $\mathbf{G}=\left[\mathbf{g}_{1}, \cdots, \mathbf{g}_{L}\right] \epsilon$ $\mathbb{C}^{N \times L}, 1 \leq L \leq M$ are lines that are perpendicular to the AT direction in each range slice, which keeps that the multiple measurement vectors $\mathbf{S}=\left[\mathbf{s}_{1}, \cdots, \mathbf{s}_{L}\right] \in \mathbb{C}^{N \times L}$ share the same
TABLE 1: System parameters.

\begin{tabular}{lc}
\hline Carry frequency & $37.5 \mathrm{GHz}$ \\
\hline Bandwidth & $300 \mathrm{MHz}$ \\
Pulse width & $4 \mu \mathrm{s}$ \\
Height of flight & $1000 \mathrm{~m}$ \\
Range sampling number & 1600 \\
AT/CT sampling interval & $0.01 \mathrm{~m}$ \\
AT/CT sampling number & 256 \\
\hline
\end{tabular}

sparse structure. Since the downsampling matrix $\Phi_{m} \in$ $\mathbb{R}^{N^{\prime} \times N}$ is fixed, it is the same for $\mathbf{s}_{1}, \cdots, \mathbf{s}_{L}$. As a result, we can express the downsampling matrix $\boldsymbol{\Phi}_{m}$ as $\boldsymbol{\Phi}$ for $m=1$, $2, \cdots, L$. The downsampling signal can be denoted as $\boldsymbol{\Xi}=$ $\boldsymbol{\Phi S}$. As a result, the $\mathbf{G}=\left[\mathbf{g}_{1}, \cdots, \mathbf{g}_{L}\right]$ can be obtained by solving the following MMV model:

$$
\Xi=\Phi S=\Phi \Psi \mathrm{G}=\mathrm{AG}
$$




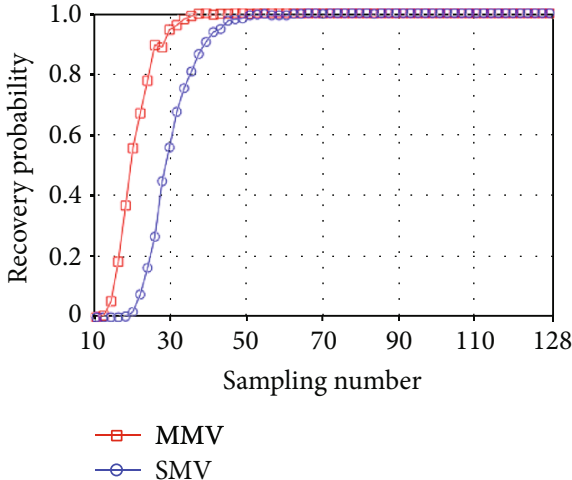

(a)

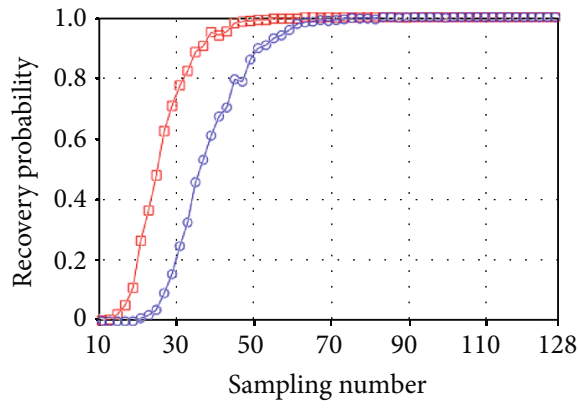

(b)

FIgURE 4: The relation of the recovery probability and the CT sampling number. (a) The number of scatters is 5. (b) The number of scatters is 10 .

Then, the CT compression needs to solve the MMV model $M / L$ times. The optimization problem of (10) can be described as

$$
\begin{aligned}
& \qquad \widehat{\mathbf{G}}=\min _{\mathbf{G}} \mathrm{F}(\mathbf{G}) \\
& \text { s.t. }\|\boldsymbol{\Xi}-\mathbf{A G}\|_{F}^{2}<\varepsilon,
\end{aligned}
$$

where $\|\cdot\|_{F}$ is the Frobenius norm, $\varepsilon$ is a constant value related with noise, and $\mathrm{F}(\mathbf{G})$ is the regularization term related to the prior distribution of $\mathbf{G}$. To obtain better robustness, we introduced a mixed $L_{2,1}$-norm for matrices to calculate the sparsity of $\mathbf{G}$. The mixed $L_{2,1}$-norm for $\mathbf{G}$ can be expressed as

$$
\left\|\mathbf{G}_{2,1}=\right\|\left[\begin{array}{c}
\left\|\mathbf{G}_{1}\right\|_{2} \\
\left\|\mathbf{G}_{2}\right\|_{2} \\
\vdots \\
\left\|\mathbf{G}_{Q}\right\|_{2}
\end{array}\right] \|_{1}=\sum_{i=1}^{Q} \sqrt{\sum_{j=1}^{L}\left|g_{i j}\right|^{2}},
$$

where $\mathbf{G}_{i}$ denote the $i$ th row of $\mathbf{G},\|\cdot\|_{2}$ denotes the $l_{2}$-norm for vectors, and $g_{i j}$ is the element of $\mathbf{G}$ at the $i$ th row and $j$ th column. In Equation (12), the $l_{2}$-norms of the rows in $\mathbf{G}$ are calculated firstly to form a column vector, and then, the $l_{1}$-norm of the formed column vector is calculated as the mixed $L_{2,1}$-norm of $\mathbf{G}$. Then, Equation (11) can be expressed as

$$
\begin{aligned}
& \qquad \widehat{\mathbf{G}}=\min _{\mathbf{G}}\|\mathbf{G}\|_{2,1} \\
& \text { s.t. }\|\boldsymbol{\Xi}-\mathbf{A} \mathbf{G}\|_{F}^{2}<\varepsilon .
\end{aligned}
$$

Utilizing the Lagrangian method, Equation (13) can be expressed as a regularization model

$$
\widehat{\mathbf{G}}=\underset{\mathbf{G}}{\arg \min } \mathbf{J}(\mathbf{G}), \quad \mathbf{J}(\mathbf{G})=\|\boldsymbol{\Xi}-\mathbf{A G}\|_{F}^{2}+\lambda\|\mathbf{G}\|_{2,1},
$$

where $\mathrm{J}(\mathbf{G})$ is the objective function and $\lambda$ is the regularization parameter. According to the regularization theory [25], the regularization parameter can be $\lambda=2 \beta^{2}$, where $\beta$ is the variance of the reconstructed error. To solve Equation (14), the iterative formula of step (3) in Algorithm 1 should be modified. As a result, we derived the gradient of the objective function $\mathrm{J}(\mathbf{G})$ as

$$
\nabla_{\mathbf{G}} \mathrm{J}(\mathbf{G})=2 \mathbf{A}^{\mathrm{H}} \mathbf{A G}-2 \mathbf{A}^{\mathrm{H}} \boldsymbol{\Xi}+\lambda \mathbf{\Lambda}(\mathbf{G}) \mathbf{G},
$$

where $\boldsymbol{\Lambda}(\mathbf{G})=\operatorname{diag}\left(1 /\left\|\mathbf{G}_{1}\right\|_{2}, 1 /\left\|\mathbf{G}_{2}\right\|_{2}, \cdots, 1 /\left\|\mathbf{G}_{Q}\right\|_{2}\right)$. The $k$ th iterative formula of $\mathbf{G}$ can be expressed as

$$
\mathbf{G}^{(k+1)}=\left(\mathbf{A}^{\mathrm{H}} \mathbf{A}+\frac{\lambda}{2} \boldsymbol{\Lambda}\left(\mathbf{G}^{(k)}\right)\right)^{-1} \mathbf{A}^{\mathrm{H}} \mathbf{\Xi} .
$$

By modifying step (3) in Algorithm 1 as (16), the MMV-OMP algorithm based on the $L_{2,1}$-norm is shown in Algorithm 2.

As shown in Figure 3, the signal after CT compression can be written as

$$
\begin{aligned}
S_{c}\left(t, t_{m}, y_{q}\right)= & \sum_{i=1}^{K} \operatorname{sinc}\left(B_{r}\left(t-\frac{2 R_{0}}{c}\right)\right) \varsigma\left(y_{q}\right) \\
& \cdot \exp \left(-j \frac{2 \pi}{\lambda} \frac{\left(v t_{m}-x_{k}\right)^{2}}{R_{0}}\right) \exp \left(-j \frac{4 \pi}{\lambda} R_{0}\right),
\end{aligned}
$$

where $\varsigma\left(y_{q}\right)$ is the result of CT compression.

3.4. Along-Track Compression. Finally, the AT compression is the same as that of the traditional methods. It is realized by the matched filtering in the AT domain, and the detailed 


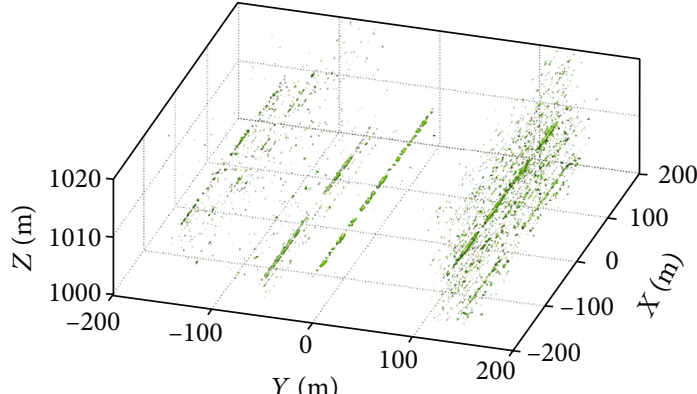

(a)

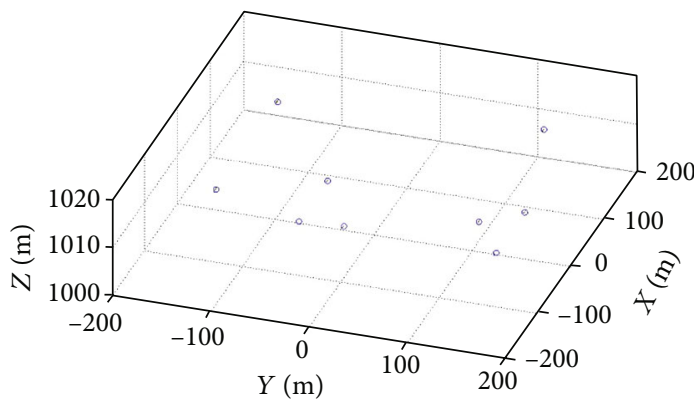

(c)

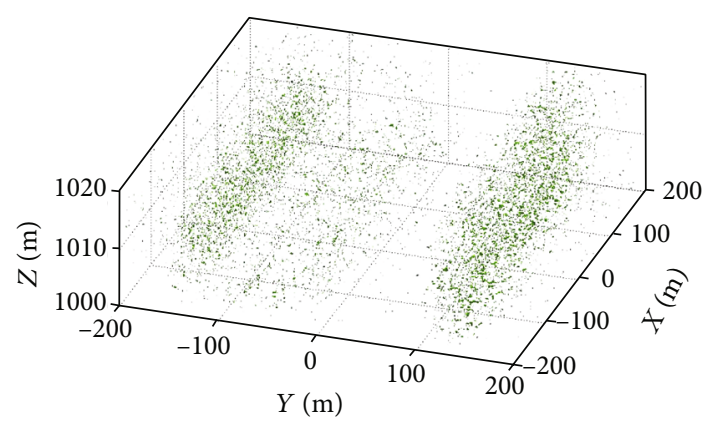

(b)

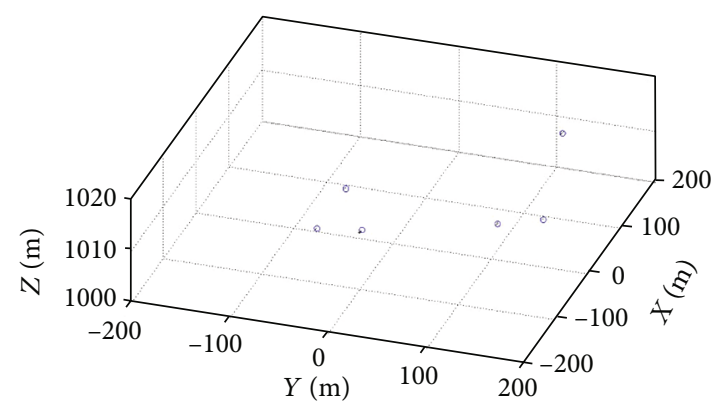

(d)

FIGURE 5: Imaging results of point targets when SNR is $-5 \mathrm{~dB}$ and $L=128$ : (a) the imaging result after the range compression and MMV-based CT compression; (b) the imaging result after the range compression and SMV-based CT compression; (c) AT compression result of (a); (d) AT compression result of (b).

information can be obtained in $[13,25]$. The 3-D imaging result is

$$
\begin{aligned}
S_{\text {final }}\left(t, t_{m}, y_{q}\right)= & \sum_{k=1}^{K} \operatorname{sinc}\left(T_{r} K_{r}\left(t-\frac{2 R_{0}}{c}\right)\right) \varsigma\left(y_{q}\right) \\
& \cdot \operatorname{sinc}\left(B_{a}\left(v t_{m}-x_{k}\right)\right) \exp \left(-j \frac{4 \pi}{\lambda} R_{0}\right),
\end{aligned}
$$

where $B_{a}$ is the AT bandwidth.

\section{Results and Discussion}

The simulations of point targets and distributed targets are given to verify the performance of the proposed algorithm. The simulations of point targets and the experiments of distributed targets utilize the same system parameters, which are chosen according to $[10,19,25]$ and shown in Table 1 .

4.1. Reconstruction Performance for Point Targets. At first, we discuss the relationship between the recovery probability, sampling number, and sparsity. We define the condition of one successful reconstruction which is

$$
\sum_{i=i}^{N_{r}} \sum_{m=1}^{M} \sum_{q=1}^{Q} \frac{\left|\sigma \wedge_{i m q}-\sigma_{i m q}\right|^{2}}{\left|\sigma_{i m q}\right|^{2}}<0.1 \text {, }
$$

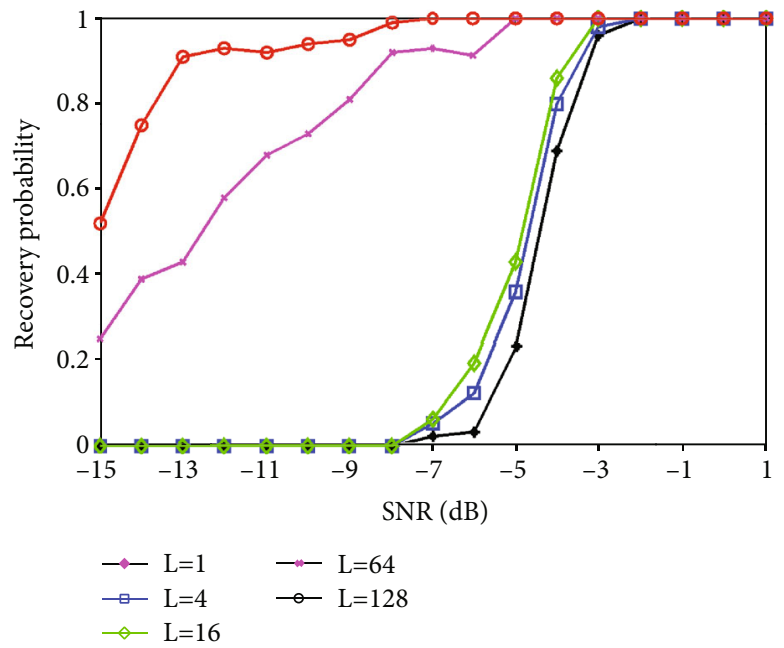

FIgURE 6: The relation of the recovery probability, SNR, and the multimeasurement number.

where $\widehat{\sigma}_{i m q}$ and $\sigma_{i m q}$ are the value of the imaging result and the scattering coefficient of the $i$ th range slice, $m$ th slow time, and the qth CT grid, respectively.

In this simulation, the range position and the AT position of the scattering points are $500 \mathrm{~m}$ and $10 \mathrm{~m}$, the CT sampling number is from 10 to 128 with interval $2, L$ is 10 , and the CT positions of the scattering points are randomly generated. In the 100 Monte Carlo trials, the recovery probability is equal to the successful reconstruction times. The relation of the recovery probability and the CT sampling 


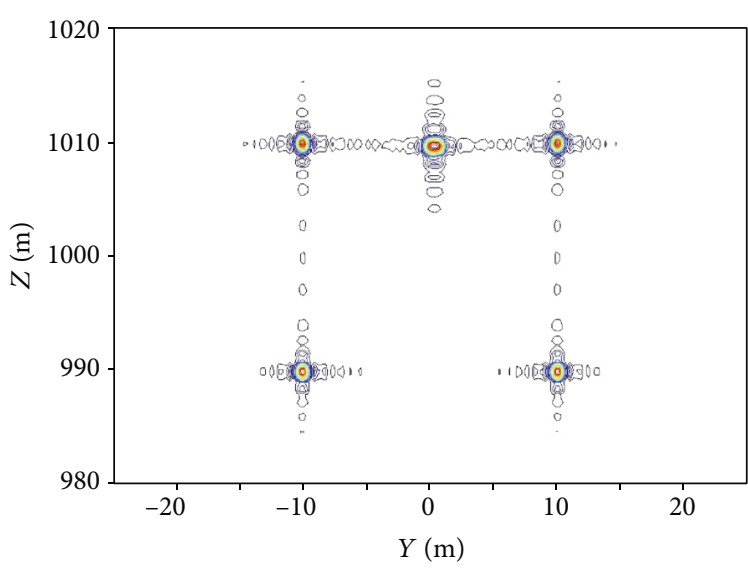

(a)

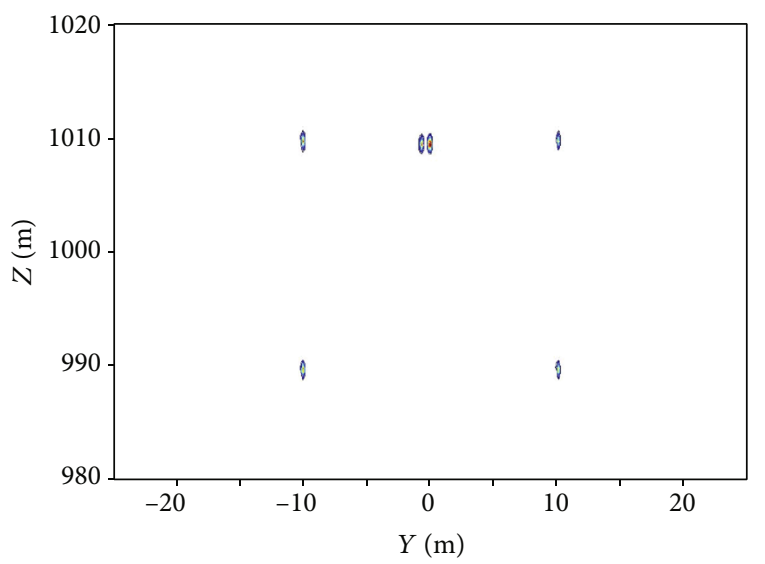

(c)

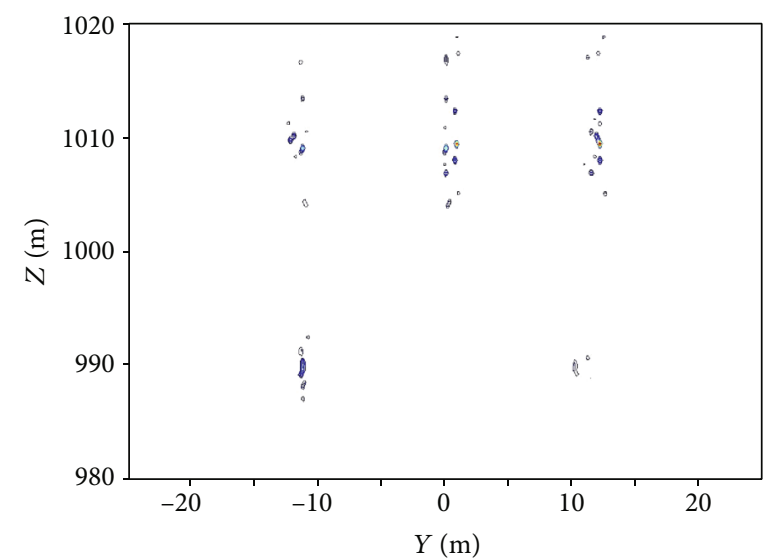

(b)

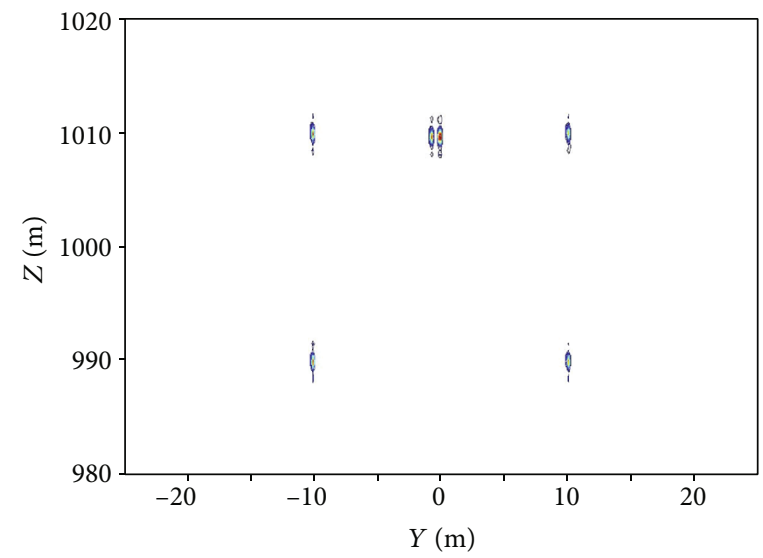

(d)

FIGURE 7: The imaging results of the 6 scattering points on the range-CT plane: (a) 3-D RD method when SNR is -5 dB, (b) SMV-based method when SNR is $0 \mathrm{~dB}$, (c) SMV-based method when SNR is $-5 \mathrm{~dB}$, and (d) the proposed MMV-based method when SNR is $-5 \mathrm{~dB}$ and $L=128$.

number is shown in Figure 4, where (a) is for 5 scattering points and (b) is for 10 scattering points. Compared with the proposed algorithm, recovery probability can achieve successful reconstruction (the recovery probability is close to 1) with a lower sampling number. The reason is as follows: Suppose the recovery probability of one CT vector by Algorithm 1 is $p$, the recovery probability of $L$ CT vectors by Algorithm 1 is $p^{L}$. As a result, the expected accurate recovery number of the CT vectors is $p \cdot L$. For Algorithm 2, since the $L C T$ vectors are regarded as a whole to calculate the support set, the recovery probability of $L$ CT vectors by Algorithm 2 is $p \cdot L / L=p \geq p^{L}$, which is higher than that of Algorithm 1. Moreover, the sampling number of successful reconstructions increases with the number of scattering points, which is due to the decrease of the sparsity.

Then, we add the white Gaussian noise into the echo signal. When SNR is $-5 \mathrm{~dB}$ and $L=128$, the imaging results of 9 random scattering points by the proposed imaging procedure are shown in Figure 5. Figures 5(a) and 5(b) are the results after MMV-based CT compression and SMV-based CT compression, respectively. Based on Figures 5(a) and 5(b), Figures 5(c) and 5(d) accomplished the AT compression, respectively. The reconstructed scattering points are marked with a blue circle in Figures 5(c) and 5(d). In Figure 5(b), the signal has been involved by the noise so that 3 scattering points are lost in Figure 5(d). However, all scattering points have been successfully reconstructed in Figure 5(c). That is, the MMV model can enhance the antinoise ability.

Then, we discuss the relationship between the recovery probability and the multimeasurement number $L$. We calculate the recovery probability when $L=1,4,16,64$, and 128 . The range of SNR is from $-15 \mathrm{~dB}$ to $1 \mathrm{~dB}$, and the step length is $1 \mathrm{~dB}$. The relation of the recovery probability, SNR, and the multimeasurement number is shown in Figure 6. It can be seen that under the same SNR, the smaller the multimeasurement number $L$ is, the lower the recovery probability is.

To verify the superresolution performance of the proposed method, we set 6 scattering points in the same AT cell. Since the Rayleigh resolution in the CT direction is about $1.5 \mathrm{~m}$ according to the system parameters, the CT distance between the two points in the middle is $1 \mathrm{~m}$, which is less than the CT Rayleigh resolution. As a result, the CT superresolution is achieved if the two points in the middle can be separated. The imaging results of the 6 scattering points on the range-CT profile are shown in Figure 7. Figure 7(a) 


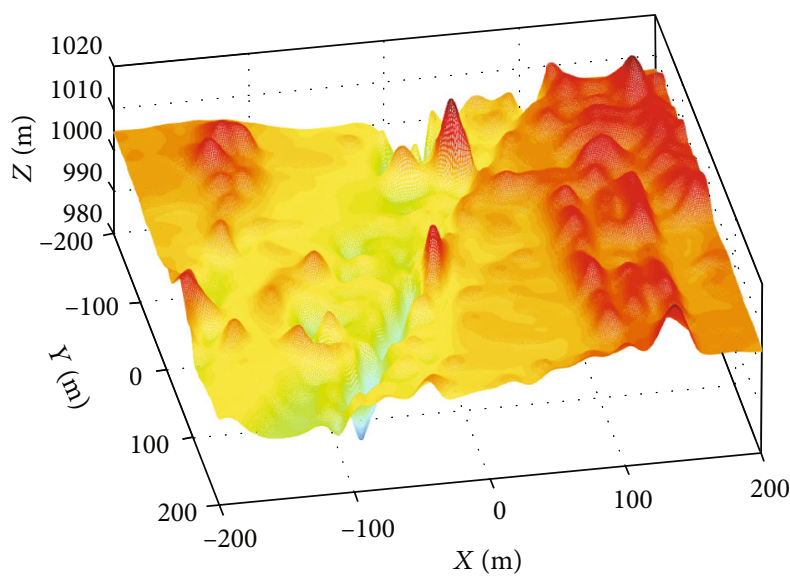

(a)

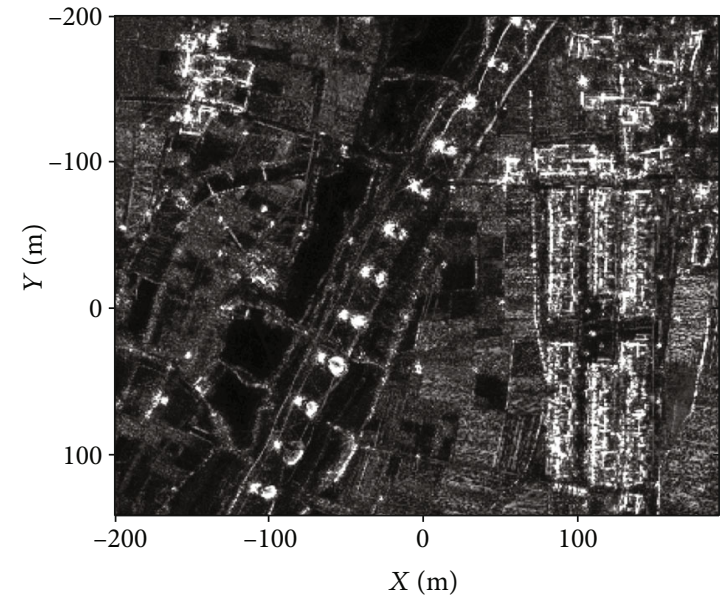

(b)

FIGURE 8: 3-D simulation scene: (a) DEM; (b) SAR image for the same region.

is obtained by the 3DRD method, in which the CT compression is realized by matched filtering so that the middle two points are overlapped. Figures 7(b) and 7(c) are obtained by the SMV-based imaging method when SNR is $-5 \mathrm{~dB}$ and $0 \mathrm{~dB}$, respectively. Although the two points in the middle can be separated in Figure 7(c), the scattering points are unfocused in Figure 7(b). As a result, the robustness of the $l_{1}$-norm-based models is unsatisfactory. Figure $7(\mathrm{~d})$ is obtained by the proposed MMV-based imaging method when SNR is $-5 \mathrm{~dB}$ and $L=128$. It can be seen that the scattering points are focused. Moreover, the two points in the middle are separated clearly, which means the proposed method can achieve the CT superresolution in the lower SNR environment.

For the SMV-OMP algorithm shown in Algorithm 1 and the MMV-OMP algorithm shown in Algorithm 2, the main computational burden includes the internal product calculation in step (1) and the matrix inversion in step (3). The comparison of computational complexity is shown in Algorithm 2. Because the range sampling number and the AT sampling number are $N_{\mathrm{r}}$ and $M$, the number of the CT vectors to be reconstructed is $M \cdot N_{\mathrm{r}}$. To obtain the imaging results, Algorithm 1 needs to run $M \cdot N_{\mathrm{r}}$ times. As a result, step (1) needs to calculate the internal product $O\left(K N_{\mathrm{r}} M N\right)$ times, and step (3) needs to calculate the matrix inversion $O\left(K N_{\mathrm{r}} M\right)$ times. Since Algorithm 2 can deal with $L$ CT vectors once, it needs to run $N_{\mathrm{r}} M / L$ times to obtain the imaging result. As a result, step (1) also needs to calculate the internal product $O\left(K N_{\mathrm{r}} M N\right)$ times, but step (3) only needs to calculate the matrix inversion $O\left(K N_{\mathrm{r}} M / L\right)$ times, where $1 \leq L \leq M$. The sparse level $K$ is positively related to the time cost. The multimeasured vector number $L$ has an important influence on the time cost.

4.2. The Experiment of Distributed Targets. In this part, we use an experiment of distributed targets to evaluate the proposed MMV-OMP-based algorithm. The simulated DLLA SAR data of distributed targets need the positions and the coefficients of scatters. The coefficients of scatters are
TABLE 2: Computation complexity.

\begin{tabular}{lcc}
\hline & SMV-OMP & MMV-OMP \\
\hline Internal product & $O\left(K N_{\mathrm{r}} M N\right)$ & $O\left(K N_{\mathrm{r}} M\right)$ \\
Matrix inversion & $O\left(K N_{\mathrm{r}} M N\right)$ & $O\left(K N_{\mathrm{r}} M / L\right)$ \\
\hline
\end{tabular}

obtained from the SAR image shown in Figure 8(b). The $\mathrm{AT}$ and CT positions of scatters are obtained from Figure $8(\mathrm{a})$. The height positions of scatters are obtained from Figure 8(b). Figures $8(\mathrm{a})$ and $8(\mathrm{~b})$ are for the same region. For the experiment of distributed 3-D targets, the above method to generate the simulated echo signal has been used in [15-19, 25]. According to the parameters in Table 2, the Rayleigh resolution is $1.5 \mathrm{~m}$. So the interval space between scatters is set as $1 \mathrm{~m}$. The scatter number in the simulation is $N_{p} N_{q}=400 \times 400$. The SNR is $-5 \mathrm{~dB}$.

As shown in Figure 9, when SNR is $-5 \mathrm{~dB}$ and the sampling rate is $25 \%$, the images of the $3-\mathrm{D}$ distributed targets obtained by 3DRD, SMV-OMP, and the proposed MMVOMP method are shown from top to bottom, respectively. Figures 9(a), 9(b), and 9(c) show the 3-D images, the $X-Y$ plane projection images, and the 3-D images colored by height. The first row of Figure 9 shows the CT compression in 3DRD realized by matched filtering. The CT ambiguity appears when the sampling rate is $25 \%$. It means that the 3DRD method cannot deal with sparse data. In the second row of Figure 9, the CT compression is realized by SMVOMP, and some scatters are out of focus when the SNR is $-5 \mathrm{~dB}$. It means that the robustness of the imaging method based on SMV-OMP is unsatisfactory in the low signal-tonoise rate (SNR) environment. In the third row of Figure 9, the CT compression is realized by the proposed MMV-OMP when $L=128$, and the imaging results focus much better than those of the SMV-OMP. It means that the proposed method can obtain better performance in the low SNR environment. 

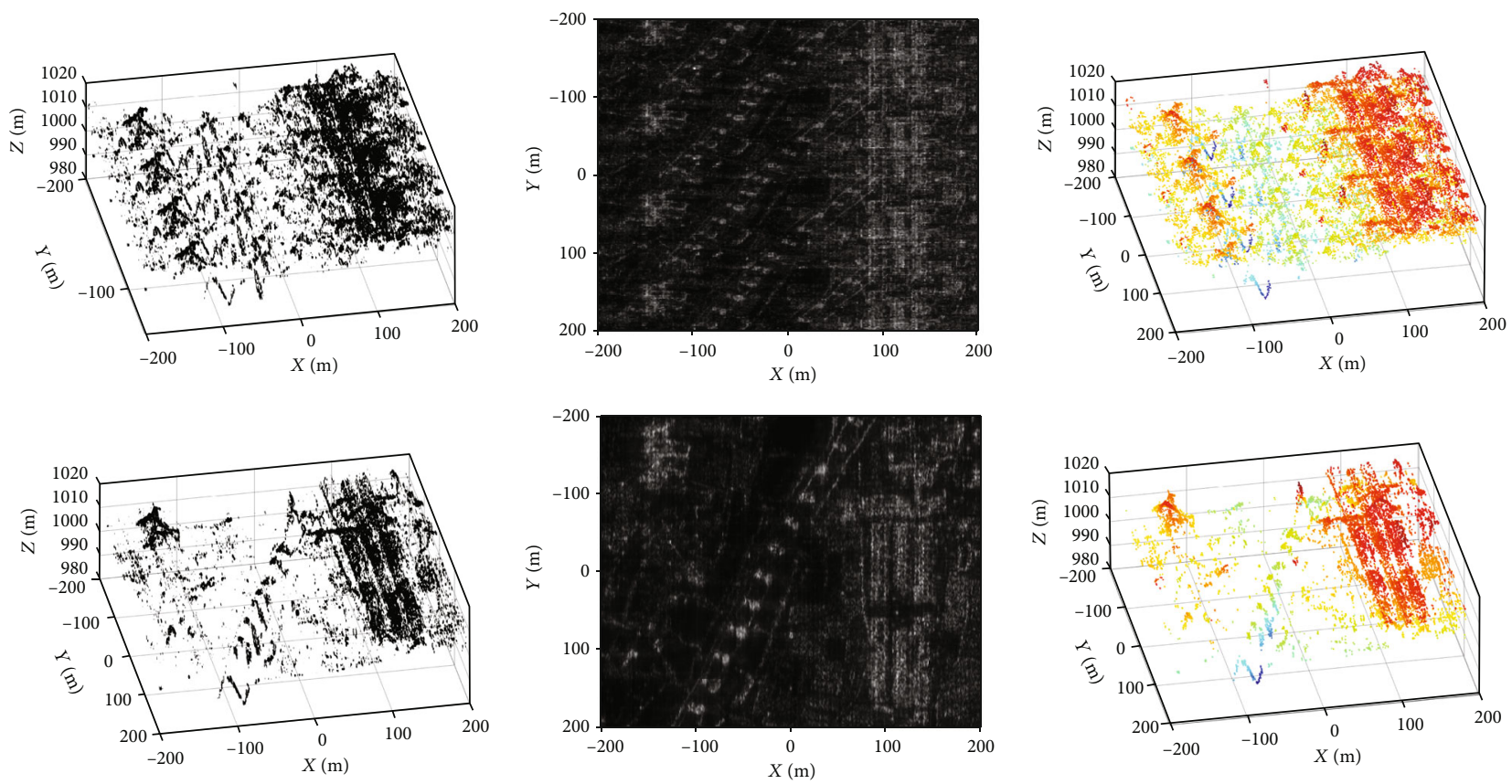

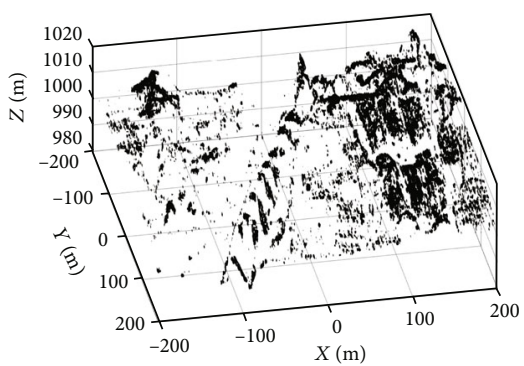

(a)

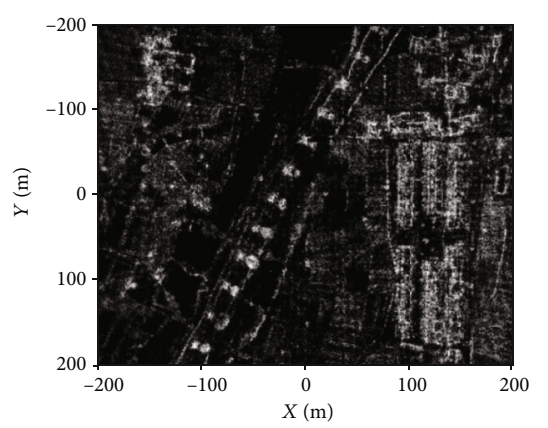

(b)

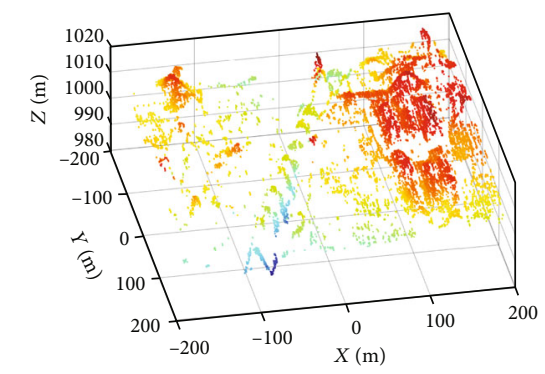

(c)

FIGURE 9: Images of the 3-D distributed targets obtained by 3DRD, SMV-OMP, and the proposed method from top to bottom when the SNR is $-5 \mathrm{~dB}$ and the sampling rate is $25 \%$ : (a) 3-D reconstructed images; (b) $X-Y$ plane projection images; (c) 3-D reconstructed images colored by height.

TABLE 3: Imaging performance of the distributed targets.

\begin{tabular}{lccr}
\hline & 3DRD & SMV-OMP & Proposed method \\
\hline Relative mean square error & 0.8734 & 0.1905 & 0.0847 \\
Mean running time (s) & $6.85 \mathrm{~s}$ & $154.72 \mathrm{~s}$ & $33.24 \mathrm{~s}$ \\
\hline
\end{tabular}

To evaluate the imaging results, the relative mean square error (RMSE) of the 3-D imaging results is defined as $\left(1 / N_{p} N_{q}\right) \sum_{k=1}^{N_{p} N_{q}}\left|\sigma \wedge_{k}-\sigma_{k}\right|^{2} /\left|\sigma_{k}\right|^{2}$. Here, $\sigma_{k}$ and $\widehat{\sigma}_{k}$ are the true value according to Figure $8(\mathrm{~b})$ and the reconstructed value of the $k$ th scatter, respectively. The RMSE of the 3-D imaging results in Figure 9(a) is shown in Table 3. The proposed method has a smaller RMSE. Table 3 shows the mean running time of the methods. The proposed method has a shorter running time than the imaging method based on SMV-OMP. As a result, the experiment of distributed targets can indicate that the proposed method has better performance in the lower SNR environment and require lower computation.

\section{Conclusions}

In this paper, we propose a novel imaging method for DLLA $3 \mathrm{D}$ SAR based on the MMV model with $L_{2,1}$-norm. Specifically, the imaging problem can be transformed into a sparse regularization model based on the MMV model since we construct the same CT sparse structure of different pulses by exchanging the processing sequence of the CT compression and the AT compression. Moreover, the mixed $L_{2,1}$ norm is introduced into the regularization term of the MMV model and the corresponding MMV-OMP algorithm is designed. The proposed method can obtain the CT superresolution in the lower SNR environment and require lower 
computation compared with the conventional methods, which is verified by the simulation experiments of point targets and distributed targets.

In the MMV model, the original vectors are required to have the same sparse structure. For the crosstrack compression in DLLA SAR, the same sparse structure in each range slice depends on the range migration correction in the crosstrack domain. As a result, the advantages of the proposed algorithm are based on the assumption of accurate range migration correction. It is our future work to solve this problem by combining the range migration correction and MMV model in DLLA SAR.

\section{Data Availability}

The simulation data used to support the findings of this study have been deposited in the GITHUB repository: https://github.com/KangLe-radarimaging/A-Novel-DLLASAR-Imaging-Method-Based-on-MMV-Model/.

\section{Conflicts of Interest}

The authors declare that there are no conflicts of interest regarding the publication of this paper.

\section{Authors' Contributions}

The author Tianchi Sun and the author Le Kang contributed equally to this work.

\section{Acknowledgments}

The authors would like to thank Professor Hong Wen of Aerospace Information Research Institute, Chinese Academy of Sciences, for sharing the experimental data in this paper. This work was supported in part by the National Natural Science Foundation of China under Grant 61631019, 61971434, and 62001508 and the Natural Science Foundation of Shanxi Province under Grant 2020JQ-480.

\section{References}

[1] X. X. Zhu, S. Montazeri, M. Ali et al., "Deep learning meets SAR: concepts, models, pitfalls, and perspectives," IEEE Geoscience and Remote Sensing Magazine, 2021.

[2] M. Soumekh, Synthetic Aperture Radar Signal Processing with Matlab Algorithms, Wiley, New York, NY, USA, 1999.

[3] E. Aguilera, M. Nannini, and A. Reigber, "Wavelet-based compressed sensing for SAR tomography of forested areas," IEEE Transactions on Geoscience and Remote Sensing, vol. 51, no. 12, pp. 5283-5295, 2013.

[4] Y. Lin, W. Hong, W. X. Tan, and Y. P. Wang, "Airborne circular SAR imaging: results at P-band," in Proceedings of the IEEE International Geoscience and Remote Sensing Symposium (IGARSS'12), pp. 5594-5597, Munich, July 2012.

[5] C. H. Gierull, "On a concept for an airborne downwardlooking imaging radar," AEU-International Journal of Electronics and Communications, vol. 53, no. 6, pp. 295-304, 1999.

[6] J. Klare, D. Cerutti-Maori, A. Brenner, and J. Ender, "Image quality analysis of the vibrating sparse MIMO antenna array of the airborne 3D imaging radar ARTINO," in 2007 IEEE International Geoscience and Remote Sensing Symposium, pp. 5310-5314, Barcelona, Spain, July 2007.

[7] J. F. Nouvel, H. Jeuland, G. Bonin, S. Roques, O. Du Plessis, and J. Peyret, "A Ka band imaging radar: DRIVE on board ONERA motorglider," in Proceedings of the IEEE International Geoscience and Remote Sensing Symposium (IGARSS'06), pp. 134-136, Denver, Colo, USA, 2006.

[8] L. Du, Y. P. Wang, and W. Hong, "A three-dimensional range migration algorithm for downward-looking 3-D SAR with single-transmitting and multiple-receiving linear array antennas," EURASIP Journal on Advances in Signal Processing, vol. 2010, no. 1, Article ID 957916, 15 pages, 2010.

[9] D. H. Zhang and X. L. Zhang, "Downward-looking 3-D linear array SAR imaging based on chirp scaling algorithm," in Proceedings of the 2nd Asian-Pacific Conference on Synthetic Aperture Radar (APSAR'09), pp. 1043-1046, Xi'an China, October 2009.

[10] X. M. Peng, W. Hong, and Y. P. Wang, "Polar format imaging algorithm with wave-front curvature phase error compensation for airborne DLSLA three-dimensional SAR," IEEE Geoscience and Remote Sensing Letters, vol. 11, no. 6, pp. 1036-1040, 2014.

[11] D. L. Donoho, "Compressed sensing," IEEE Transactions on Information Theory, vol. 52, no. 4, pp. 1289-1306, 2006.

[12] E. J. Candes, J. Romberg, and T. Tao, "Robust uncertainty principles: exact signal reconstruction from highly incomplete frequency information," IEEE Transactions on Information Theory, vol. 52, no. 2, pp. 489-509, 2006.

[13] X. Ren, L. Chen, and J. Yang, “3-D imaging algorithm for down-looking MIMO array SAR based on bayesian compressive sensing," International Journal of Antennas \& Propagation, vol. 2014, article 612326, 2014.

[14] S. Q. Zhang, Y. T. Zhu, G. G. Dong, and G. Y. Kuang, "Truncated SVD-Based compressive sensing for downward-looking three-dimensional SAR imaging with uniform/nonuniform linear array," IEEE Geoscience and Remote Sensing Letters, vol. 12, no. 9, pp. 1853-1857, 2015.

[15] X. M. Peng, W. X. Tan, W. Hong, C. Jiang, Q. Bao, and Y. Wang, "Airborne DLSLA 3-D SAR image reconstruction by combination of polar formatting and <inline-formula $>$ $<$ tex-math notation="LaTeX" $>\$$ L_ $1 \$</$ tex-math $><$ /inlineformula $>$ regularization," IEEE Transactions on Geoscience and Remote Sensing, vol. 54, no. 1, pp. 213-226, 2016.

[16] Q. Bao, K. Y. Han, L. Yun, B. C. Zhang, J. G. Liu, and W. Hong, "Imaging method for downward-looking sparse linear array three-dimensional synthetic aperture radar based on reweighted atomic norm," Journal of Applied Remote Sensing, vol. 10, no. 1, article 015008, 2016.

[17] Q. Bao, K. Y. Han, X. M. Peng, W. Hong, B. C. Zhang, and W. X. Tan, "DLSLA 3-D SAR imaging algorithm for off-grid targets based on pseudo-polar formatting and atomic norm minimization," Science China Information Sciences, vol. 59, no. 6, article 062310, 2016.

[18] Q. Bao, X. M. Peng, Z. R. Wang, Y. Lin, and W. Hong, "DLSLA 3-D SAR imaging based on reweighted gridless sparse recovery method," IEEE Geoscience and Remote Sensing Letters, vol. 13, no. 6, pp. 841-845, 2016.

[19] Q. Bao, C. L. Jiang, L. Yun, W. X. Tan, Z. R. Wang, and W. Hong, "Measurement matrix optimization and mismatch problem compensation for DLSLA 3-D SAR cross-track reconstruction," Sensors, vol. 16, no. 8, pp. 1333-1349, 2016. 
[20] J. Chen and X. Huo, "Theoretical results on sparse representations of multiple-measurement vectors," IEEE Transactions on Signal Processing, vol. 54, no. 12, pp. 4634-4643, 2006.

[21] Y. Chen, Q. Zhang, Y. A. Chen, and L. Sun, "Modified multiple measurement vectors model for squinted synthetic aperture radar imaging," Journal of Electronic Imaging, vol. 26, no. 3, p. 033026, 2017.

[22] D. Y. Ao, R. Wang, C. Hu, and Y. H. Li, "A sparse SAR imaging method based on multiple measurement vectors model," Remote Sensing, vol. 9, no. 3, p. 297, 2017.

[23] Q. Y. Liu, Q. Zhang, and F. F. Gu, "Downward-looking linear array 3D SAR imaging based on multiple measurement vectors model and continuous compressive sensing," Journal of Sensors, vol. 2017, Article ID 6207828, 11 pages, 2017.

[24] Y. Shen, S. Li, Department of Mathematics, Zhejiang Sci-Tech University, Hangzhou, 310018, and Department of Mathematics, Zhejiang University, Hangzhou, 310027, "Sparse signals recovery from noisy measurements by orthogonal matching pursuit," Inverse Problems \& Imaging, vol. 9, no. 1, pp. 231238, 2015.

[25] L. Kang, T. C. Sun, Y. Luo, Q. Zhang, and J. C. Ni, “Downward-looking linear array three-dimensional SAR imaging based on the two-dimensional mismatch compensation," IEEE Journal of Selected Topics in Applied Earth Observations and Remote Sensing, vol. 14, no. 1, pp. 258-269, 2021. 